\title{
The Solar Energetic Particle Event of 6 May 1998
}

\author{
T.T. von Rosenvinge ${ }^{1}$, C.M.S. Cohen ${ }^{2}$, E.R. Christian ${ }^{1}$, A.C. Cummings ${ }^{2}$, \\ R.A. Leske ${ }^{2}$, R.A. Mewaldt ${ }^{2}$, P.L. Slocum ${ }^{3}$, E.C. Stone ${ }^{2}$, \\ and M.E. Wiedenbeck ${ }^{3}$ \\ ${ }^{1}$ NASA/Goddard Space Flight Center, Greenbelt, MD 20771 \\ ${ }^{2}$ California Institute of Technology, Pasadena, CA 91125 \\ ${ }^{3}$ Jet Propulsion Laboratory, Pasadena, CA 91109
}

\begin{abstract}
The abundances of elements from helium to iron have been measured in more than a dozen moderate to large solar energetic particle (SEP) events using the Solar Isotope Spectrometer (SIS) on-board the Advanced Composition Explorer (ACE). Time variations within some of these events and from event to event have been reported previously. This paper presents an analysis of the event of 6 May 1998, for which relatively time-independent abundance ratios are found. This event has been considered to be an example of an impulsive event, a gradual event, and as a hybrid of the two. Difficulties with classifying this event are discussed.
\end{abstract}

\section{INTRODUCTION}

Well prior to the launch of the Advanced Composition Explorer (ACE) there was an established model of solar energetic particle (SEP) events which divided events into two classes, impulsive events and gradual events $(1,2)$. In addition, it had been proposed that some events are hybrid events which combine an initial impulsive event with a gradual event $(3,4)$. Since the launch of ACE there has been considerable debate as to whether certain events are gradual, impulsive, or hybrid events. In particular, the nature of the 6 May 1998 event has been extensively debated and will be the topic of this paper.

The characteristics of impulsive and gradual events have been summarized by Reames (1). In brief, impulsive events are events which are associated with acceleration by solar flares, whereas gradual events are associated with acceleration by shocks driven by Coronal Mass Ejections (CMEs). Starting in the corona, the strongest such shocks can continue to accelerate particles all the way out to $1 \mathrm{AU}$ and beyond. Initially the terms impulsive and gradual referred to the durations of corresponding soft x-ray events, with 1 hour roughly marking the dividing line between the two. Subsequently the terms have evolved to refer to the durations of the particle events themselves, with impulsive events, at a few MeV/nuc, typically lasting for only hours, and gradual events lasting for several days (1). This of course leaves a wide window for events in between. In addition, no clear-cut criteria for measuring an event's duration have been established.

Impulsive events are typically ${ }^{3} \mathrm{He}$-rich and have composition enhanced in heavy elements relative to coronal abundances. They are also associated with Type III radio bursts and low energy electrons. By contrast, gradual events are not ${ }^{3} \mathrm{He}$-rich and are associated with Type II and Type IV radio emission. Their average abundances reflect coronal abundances (5). Mean Fe charge states were initially measured at $\sim 1 \mathrm{MeV} /$ nuc to be $20.5 \pm 1.2$ in impulsive events and $14 \pm 1$ in gradual events (6). Recent measurements have shown that 14 is perhaps an intermediate value (7), with some gradual events having a mean Fe charge state as low as $11(7,8)$. The relationship between these intermediate events and hybrid events, if any, has not been established. The charge states in gradual events are also, at least on occasion, energy dependent $(9,10,11)$.

Due to the large scale of CMEs and their associated shocks, gradual event particles appear on magnetic field lines which connect back to the sun over a wide range of solar longitudes. By comparison, an observer must be well-connected magnetically to the flare site on the sun to observe an impulsive event. The observed range of good connection longitudes is from $\sim 30-80^{\circ}$ West (2).

Historically, events have been considered to be ${ }^{3} \mathrm{He}$-rich when ${ }^{3} \mathrm{He} /{ }^{4} \mathrm{He}$ exceeded $10 \%$, but this was largely due to the fact that early instruments could not reliably observe ${ }^{3} \mathrm{He} /{ }^{4} \mathrm{He}$ below about $10 \%$. With improved measurement capabilities on ACE, it is apparent that many events have ${ }^{3} \mathrm{He} /{ }^{4} \mathrm{He}$ ratios which 
are below $10 \%$ and yet are much enhanced over the average solar wind value of $\sim 0.04 \%$.

The Solar Isotope Spectrometer (SIS) on-board the Advanced Composition Explorer (ACE) has a large geometry factor $\left(\sim 40 \mathrm{~cm}^{2}-\mathrm{sr}\right)$, enabling abundances in solar energetic particle (SEP) events to be observed on a time scale of hours or less. Substantial temporal variations of abundances from event to event and within events have been reported earlier using SIS data (12). For example, $\mathrm{Fe} / \mathrm{O}$ varied from as low as $\sim 0.1$ times the average $\mathrm{Fe} / \mathrm{O}$ value for gradual events to as high as $\sim 8$ times the average value in the events of 20 April 1998 and 6 November 1997, respectively. He/O varied from $\sim 2$ times average to $\sim 0.1$ times average within the 20 April 1998 event. (The average values for gradual events obtained from (5) are used as convenient reference levels for identifying enhancements and depletions). $\mathrm{Ca} / \mathrm{O}$ enhancements are frequently similar to $\mathrm{Fe} / \mathrm{O}$ enhancements. $\mathrm{Si} / \mathrm{O}$ is rarely enhanced, although the event of 14 November 1998 had Si/O enhanced by $\sim 2$ times average. Time histories of the intensities and abundance ratios in these events are shown in (12).

Besides events with large temporal variations, some events have abundances which are essentially time-independent for all elements. The first four such events observed by SIS (6 November 1997, 2 May 1998, 6 May 1998, and 14 November 1998) all had abundances similar to the average abundances of impulsive events. These abundances are unlike the average abundances of gradual events $(13,12)$. However, other analyses (Tylka and Reames, private communication; also (14)) have led to the conclusion that all four of these events were gradual events. It has also been suggested that one of these events, the event of 6 May 1998, is a hybrid of an impulsive and a gradual event (15).

Ultimately it is essential to understand the temporal variations of solar energetic particle abundances in order to understand particle acceleration mechanisms and to reliably estimate the composition of the sun from direct observations of SEP abundances. To do so, each event needs to be separately understandable. The event studied here is sufficiently complex to challenge current thinking.

\section{OBSERVATIONS}

Solar observations for this period are given in NOAA's Solar-Geophysical Data Reports (16). There were two solar X-ray events, M class or greater, on 6 May 1998, both in a region nominally located at S11W65. The first of these, a class M2.9 event, started at 07:10, peaked at 07:25, and ended at 07:41. The second event, of class $\mathrm{X} 2.7$, started at 07:58, peaked at
08:09, and ended at 08:20. Type III emission started at $07: 35$ and continued intermittently to 08:13. Type II and IV radio emission started at 08:00 and at 08:03, respectively, presumably due to the second event. A CME was first observed in the LASCO C2 field of view (1.5-6 solar radii) at 08:04. The speed of the CME was measured to be $1053 \mathrm{~km} / \mathrm{sec}(17)$. This CME is clearly associated with the second $\mathrm{x}$-ray event and not the first. Type III emission is clearly associated with the first $\mathrm{x}$ ray event and possibly with the second one as well.

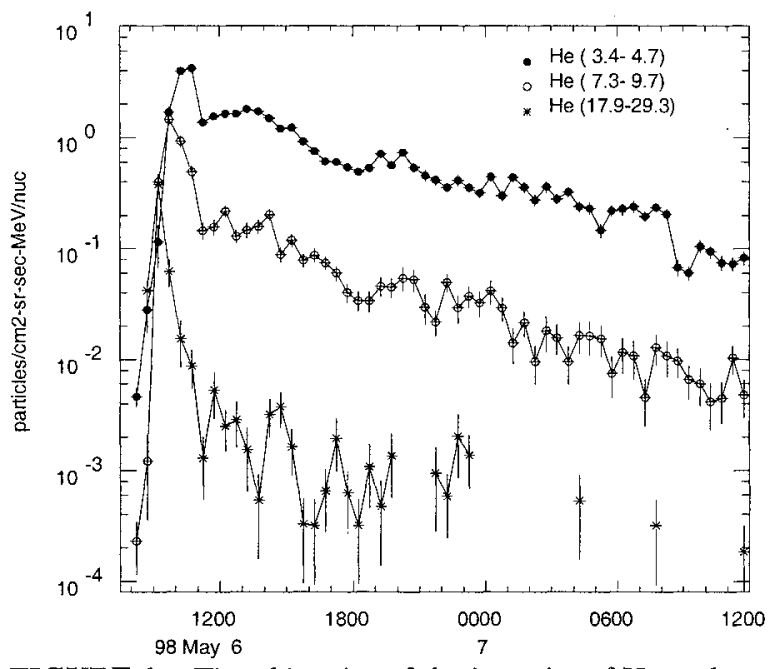

FIGURE 1. Time histories of the intensity of He at three different energies for the event of 6 May 1998.

Figure 1 shows the time history of He for this event at three different energies. The intensity of $\mathrm{He}$ in the energy interval 17.9 to $29.3 \mathrm{MeV} /$ nuc fell by one and a half orders of magnitude in just over 2 hours and then declined further at a much slower rate. He in the energy interval 3.4 to $4.7 \mathrm{MeV} /$ nuc shows a relatively gradual decline, with some evidence of the initial spike evident at higher energies. The latter is an unusual feature not present in the other events observed by SIS. Reames et al. (14) saw no sign of an initial spike in this event at low energies. Surprisingly, they also saw no evidence for a spike for $\mathrm{H}$ at $20 \mathrm{MeV} / \mathrm{n}$.

The top panel of Figure 2 shows intensity-time histories for ${ }^{4} \mathrm{He},{ }^{3} \mathrm{He}$, and $\mathrm{O}$ all at $10.5 \mathrm{MeV} / \mathrm{nuc}$. The middle panel shows the corresponding ratio of ${ }^{3} \mathrm{He} /{ }^{4} \mathrm{He}$, while the bottom panel shows the ratio of ${ }^{4} \mathrm{He} / \mathrm{O}$ normalized to the gradual event average value of ${ }^{4} \mathrm{He} / \mathrm{O}$. SIS abundances have been analyzed previously at 14 $\mathrm{MeV} /$ nuc because the energy ranges of the SIS instrument are different for different elements; 14 $\mathrm{MeV} / \mathrm{nuc}$ is the lowest energy that intensities are available for every element from $\mathrm{He}$ to $\mathrm{Ni}$. Figure 2 corresponds to longer time averages than used in Figure 1 and somewhat lower energy than $14 \mathrm{MeV} /$ nuc in order to improve the statistics for ${ }^{3} \mathrm{He}$.

From the middle panel of Figure 2, the value of ${ }^{3} \mathrm{He} /{ }^{4} \mathrm{He}$ at the impulsive peak is the same as the value 

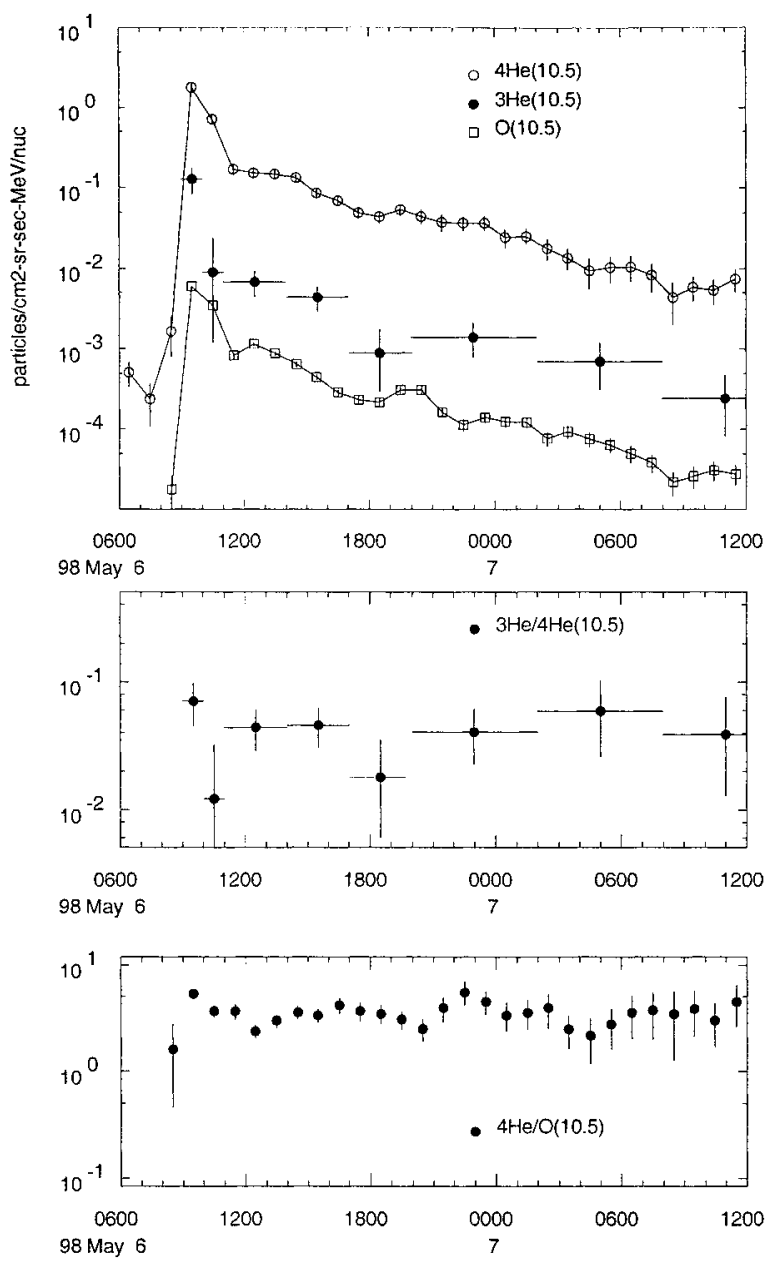

FIGURE 2. Top: 1-hour resolution time history profiles for ${ }^{4} \mathrm{He},{ }^{3} \mathrm{He}$, and $\mathrm{O}$ at $10.5 \mathrm{MeV} /$ nuc. Middle: ${ }^{3} \mathrm{He} /{ }^{4} \mathrm{He}$ versus time for $10.5 \mathrm{MeV} / \mathrm{nuc}$. Bottom: ${ }^{4} \mathrm{He} / \mathrm{O}$ versus time for $10.5 \mathrm{MeV} /$ nuc. ${ }^{4} \mathrm{He} / \mathrm{O}$ has been normalized to the average gradual event value from (5).

of subsequent points during the slow decay phase of the event. The value of ${ }^{3} \mathrm{He} /{ }^{4} \mathrm{He}$ averaged over the event is about $5 \%$. The bottom panel of Figure 2 shows that the value of ${ }^{4} \mathrm{He} / \mathrm{O}$, while enhanced, changes relatively little during the event. Figure 3 shows the time histories of the abundance ratios $\mathrm{Mg} / \mathrm{O}, \mathrm{Si} / \mathrm{O}, \mathrm{S} / \mathrm{O}, \mathrm{Ca} / \mathrm{O}$, and $\mathrm{Fe} / \mathrm{O}$ given as two hour averages at $14 \mathrm{MeV} / \mathrm{nuc}$. The first data point of each panel coincides with the 'impulsive' peak at the leading edge of the event and subsequent data points correspond to the gradual decay phase. It is apparent that the ratios $\mathrm{S} / \mathrm{O}, \mathrm{Ca} / \mathrm{O}$, and $\mathrm{Fe} / \mathrm{O}$ are all enhanced. It is also apparent that there is essentially no change in these ratios from the initial values for at least 12 hours.
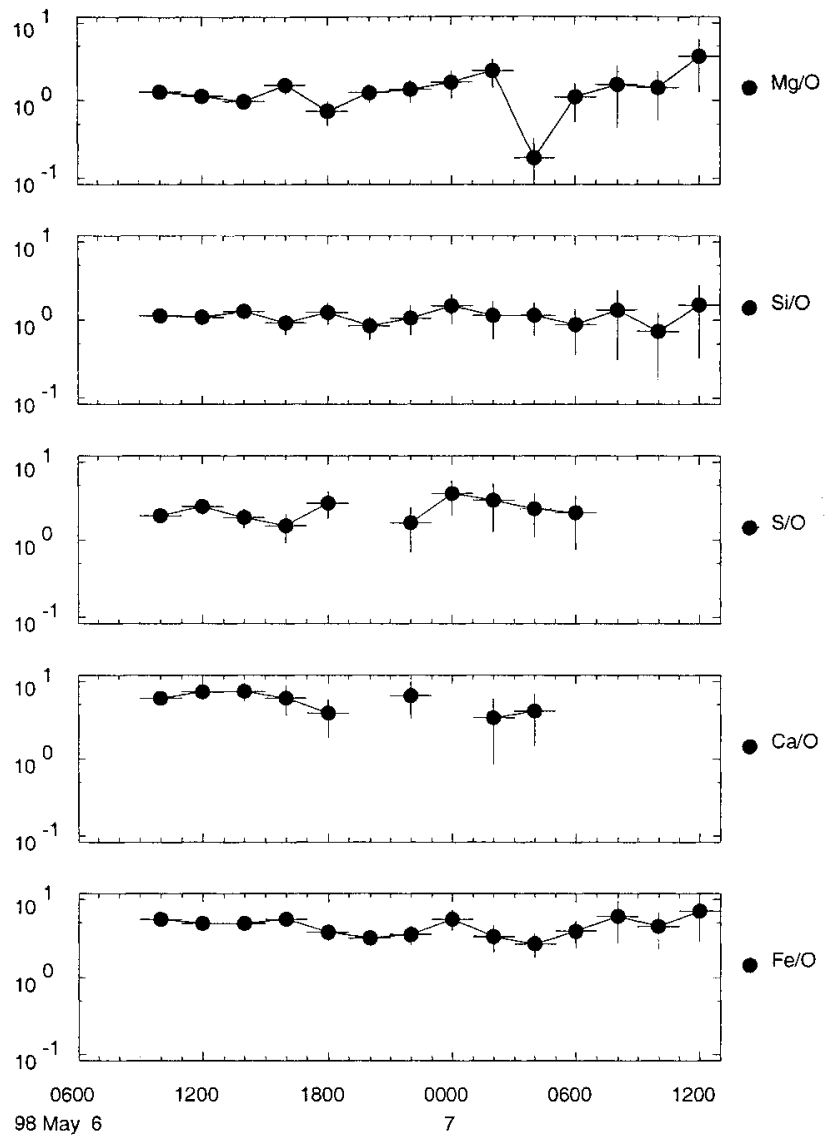

FIGURE 3. 2-hour averages of $\mathrm{Mg} / \mathrm{O}, \mathrm{Si} / \mathrm{O}, \mathrm{S} / \mathrm{O}, \mathrm{Ca} / \mathrm{O}$, and $\mathrm{Fe} / \mathrm{O}$ at $14 \mathrm{MeV} /$ nuc as a function of time for the event of 6 May 1998. These ratios are normalized to the average gradual event values from (5).

\section{DISCUSSION}

The 6 May 1998 event has been described variously as an impulsive event (13), as a gradual event (14), and as a hybrid of an impulsive event plus a gradual event (15). These interpretations are complicated by the fact that the X2.7 x-ray event was preceded by an M2.9 xray event with Type III emission. This preceding $\mathrm{x}$-ray event has been previously ignored, but it would clearly allow for yet other interpretations. For example, the first event could be impulsive and the second purely gradual. For the moment, it will be assumed that the first event can be ignored.

The 6 May 1998 event, then, appears to be impulsive because:

- the initial intensity spike has a duration of only about 2 hours

- its abundances are very similar to the average elemental abundances of impulsive events and not like those of gradual events (13). 
- the event-averaged ratio of ${ }^{3} \mathrm{He} /{ }^{4} \mathrm{He}, \sim 5 \%$, is enhanced well above the coronal value even if it doesn't quite reach the historical standard of $10 \%$.

- it is well connected to the associated flare region.

The 6 May 1998 event appears to be gradual because:

- the event profile at a few MeV/nuc looks like a normal gradual event.

- the event is associated with a fast CME.

- the event is associated with Type II and Type IV radio emission.

- there are time variations in abundances at a few $\mathrm{MeV} / \mathrm{nuc}$ (not evident at SIS energies) which can be explained well in terms of waves generated in the vicinity of the shock (14).

The suggestion that the 6 May 1998 event is a hybrid event (15) is appealing because it allows an apparent resolution to the argument as to whether the event is either impulsive or gradual. That is, it removes the need to make a choice. In addition, Popecki, et al. (15) have shown that the charge states of Fe vary during the event, being initially high (an indicator of an impulsive event) followed by lower values during the gradual phase (more consistent with the charge states normally associated with gradual events). However, the measured differences are not so large: initially the mean Fe charge state is $\sim 14.7 \pm 0.6$, then dropping to $\sim 13$ (15). Moebius, et al. (7) have classified this event as being in the previously mentioned class of intermediate Fe mean charge states.

Both the ${ }^{3} \mathrm{He} /{ }^{4} \mathrm{He}$ and the elemental abundance ratios are consistent with having the same values during both the impulsive spike and the gradual decay phase. This seems highly unlikely to occur for a hybrid event in which the gradual phase is independent of the impulsive phase. First of all, one has to ask where the enhanced ${ }^{3} \mathrm{He}$ comes from during the gradual phase. If one argues that this comes from ${ }^{3} \mathrm{He}$ left over from other, previous impulsive events (18), such as possibly the M2.9 x-ray event mentioned before, then there is no reason to expect that the value of ${ }^{3} \mathrm{He} /{ }^{4} \mathrm{He}$ from these earlier events would match that from the impulsive portion of the hybrid event. This follows since it is well known that, even with a floor of $10 \%$, the ${ }^{3} \mathrm{He} /{ }^{4} \mathrm{He}$ ratio varies by factors of 20 and more from event to event (e.g. 5). In addition, the acceleration of ${ }^{3} \mathrm{He}$ is due to a highly non-linear resonance process which is unlikely to produce the same ${ }^{3} \mathrm{He} /{ }^{4} \mathrm{He}$ ratio, even if repeating in the same region. Second of all, since the average elemental abundances are very different for impulsive and gradual events, it is difficult to see why the abundances of heavy elements shown in Figure 3 would also be so constant if the two phases were independent. One possibility then is that the event is a hybrid event but that the composition in the gradual phase is not independent of the impulsive phase. For example, perhaps the impulsive event injected particles into the coronal shock and the shock then accelerated them to SIS energies. This was suggested by (19) to explain the 3 June 1982 event. Cliver (4) proposed an alternative model, which also couples the impulsive and gradual phases. In this model, particles are first accelerated impulsively in a flare phase, populating a post-flare loop. Subsequently gradual phase particles are accelerated in the magnetic reconnection region behind the associated CME. Yet another possibility for coupling the two phases would be a single, impulsive injection that has a scatter-free initial spike with a subsequent diffusive wake (20). This could explain the common, impulsive-like composition in both the impulsive spike and the subsequent gradual decay. However, both of the latter two explanations ignore the presence of the CME driven shock. We conclude that the event could be a hybrid event, but, if so, with the two phases coupled by some as yet unknown means.

\section{ACKNOWLEDGMENTS}

This research was supported by the National Aeronautics and Space Administration at the California Institute of Technology (under grant NAG5-6912), the Goddard Space Flight Center, and the Jet Propulsion Laboratory.

\section{REFERENCES}

1. Reames, D.V., Rev. Geophys., 33, p. 585 (1995).

2. Reames, D.V., Sp. Sci. Rev., 90, p. 413 (1999).

3. Cane, H.V., et al., Ap. J., 373, p. 675 (1991).

4. Cliver, E.W., in High Energy Solar Physics, R. Ramaty, et al. (eds.), AIP (Woodbury, NY), 1995, p. 45.

5. Reames, D.V., Adv. Space Res., 15, p. 41 (1995).

6. Luhn, et al., Ap. J., 317, p. 951 (1987).

7. Moebius, et al., Proc. $26^{\text {th }}$ Int. Cosmic Ray Conf. (Salt Lake City), 6, 1999, p. 87.

8. Mason, G.M., et al., Ap. J., 452, p. 901 (1995).

9. Oetliker, et al., Ap. J., 477, p. 495 (1997).

10. Mazur, J.E., et al., Geophys. Res. Lett., 26, p. 173 (1999).

11. Möbius, E., et al., Geophys. Res. Lett., 26, p. 145 (1999).

12. von Rosenvinge, et al., Proc. $26^{\text {th }}$ Int. Cosmic Ray Conf. (Salt Lake City), 6, 1999, p. 131.

13. Cohen, C.M.S., et al., Geophys. Res. Lett., 26, p. 2697 (1999).

14. Reames, D.V., et al., Ap. J. Lett., 531, p. L83 (2000).

15. Popecki, et al., Proc. $26^{\text {th }}$ Int. Cosmic Ray Conf. (Salt Lake City), 6, 1999, p. 187.

16. NOAA Solar-Geophysical Data Reports, \#646, Part I , p.6 and p. 30 (June, 1998); \#647, Part I, pp. 121-122 (July, 1998); \#651, Part II, p. 43 (November, 1998).

17. LASCO CME list, http://lascowww.nrl.navy.mil/cmelist.html.

18. Mason, G.M., et al., Ap. J. Lett., 525, p. L133 (1999).

19. Van Hollebeke, et al., Ap. J. Suppl., 73, p. 285 (1990).

20.Earl, J., Ap. J., 188, p. 379 (1974). 